\title{
CYBERBULLYING: DIFERENCIAS EN FUNCIÓN DEL SEXO Y CURSO ACADÉMICO
}

\author{
Alberto Javier Clemente Soriano \\ Universidad Internacional de Valencia (VIU), España \\ albertojavier.clemente@campusviu.es \\ Laura Carrascosa \\ Universidad de Valencia, Valencia, España \\ Jessica Ortega-Barón \\ Universidad Internacional de la Rioja (UNIR), Valencia, España \\ Begoña Iranzo \\ Universidad Internacional de Valencia (VIU), España
}

Fecha de Recepción: 17 Febrero 2019

Fecha de Admisión: 30 Abril 2019

\section{RESUMEN}

El cyberbullying es un problema grave y transcultural. Los estudios por lo que se refiere a la diferencia en cuanto al género y la edad de víctimas y agresores son inconsistentes. En este contexto, los objetivos del presente estudio fueron analizar si existen diferencias en la cibervictimización y ciberagresión en función del sexo (chicos y chicas), y el curso académico (1ํㅜ는 $3^{\circ}$, y $4^{\circ}$ de la ESO). Participaron 660 adolescentes pertenecientes a cuatro institutos públicos de edades comprendidas entre los 12 y los 18 años. Los resultados muestran que no existen diferencias significativas entre chicos y chicas ni en la cibervictimización, ni en la ciberagresión. Respecto al curso académico, no hay diferencias en función del curso en la cibervictimización. Sin embargo, en la ciberagresión los alumnos de $3^{\circ}$ y $4^{\circ}$ de la ESO tienen puntuaciones significativamente mayores en comparación a los alumnos de $1^{0}$ de la ESO. Estos resultados evidencian la importancia de prevenir la conducta de cyberbullying en los adolescentes. Se discuten estos resultados y sus implicaciones para futuras investigaciones.

Palabras clave: adolescentes; cyberbullying; sexo; curso académico

\section{ABSTRACT}

Cyberbullying: differences based on sex and academic course. Cyberbullying is a serious and transcultural problem. The studies regarding the difference in terms of gender and age of victims and aggressors are inconsistent. In this context, the aims of this study were to analyze if there are differences in cyber-victimization and cyber-aggression according to sex (boys and girls), and the academic year (1st, 2nd, 3rd, and 4th of ESO). The sample was composed by 660 adolescents aged 


\section{CYBERBULLYING: DIFERENCIAS EN FUNCIÓN DEL SEXO Y CURSO ACADÉMICO}

between 12 and 18 from four high schools. The results show that there are no significant differences between boys and girls in cybervictimization and cyber-aggression. Regarding the academic year, there are no differences depending on the grade in cybervictimization. However, in cyber-aggression, students in 3rd and 4th grade have significantly higher scores compared to students in the 1st grade. These results demonstrate the importance of preventing cyberbullying behavior in adolescents. These results and their implications for future research are discussed.

Keywords: teenagers; cyber-bullying; sex; academic year

\section{INTRODUCCIÓN}

Los avances tecnológicos en estos últimos años han propiciado la irrupción de las tecnologías de la información y la comunicación (en adelante TIC) que han supuesto un cambio significativo en las relaciones personales, debido a la gran cantidad de contenidos en red y a la inmediatez con la que se pueden adquirir (Rodríguez-Álvarez, Cabrera y Yubero, 2018). El uso de estas tecnologías por parte de los jóvenes ha aumentado exponencialmente en los últimos años (Betts y Spencer, 2017).

El uso de las TIC es una gran ventaja para los más jóvenes, pero también, a edades cada vez más tempranas, puede suponer un mayor riesgo de uso inapropiado (Ortega-Barón, Postigo, Iranzo, Buelga y Carrascosa, 2018). Entre estos usos dañinos e inapropiados, a partir del año 2000, se comienza a conceptualizar y estudiar una conducta intencional y dañina que utiliza las TIC, el ciberbullying (Aboujaoude, Savege, Starcevic y Salame, 2015; Smith, 2019). El ciberbullying es un área de estudio de creciente interés (Smith, 2019, Zych, Ortega y Marín López, 2016), el cual se percibe como un serio problema para padres y educadores (Garaigordóbil, 2011; Pachin e Hinduja, 2013), y se considera como una "amenaza grave de salud pública" (Aboujaoude et al., 2015 p. 10).

Se define el ciberbullying como "un acto agresivo e intencional llevado a cabo por un grupo 0 un individuo, utilizando formas electrónicas de contacto, repetidamente y en el tiempo contra una víctima que no puede defenderse fácilmente." (Smith, Mahdavi, Carvalho, Fisher, Russell y Tippet 2008, p. 376). Esta definición, ha sido propuesta en base a la definición clásica de bullying dad por Olweus (1998).

Sin embargo, en el ciberbullying encontramos unas características que posibilitan que este tipo de ciberconducta pueda ser potencialmente más dañina para todos los involucrados, especialmente para las víctimas (Giumetti y Kowalsky, 2016, Ortega-Barón y Carrascosa, 2018). En primer lugar, es un tipo de acoso indirecto que puede favorecer el anonimato y facilitar una disminución de respuestas de empatía y desconexión moral en los ciberagresores (Garaigordóbil y Martínez Valderrey, 2014; Smith, 2013). En segundo lugar, las conductas de ciberbullying llegan a una mayor audiencia entre el grupo de iguales en comparación a la reducida audiencia de los comportamientos de acoso en el bullying tradicional (Garaigordóbil y Martínez Valderrey, 2014); por último como tercera característica diferencial, las y los adolescentes ciberacosados, no pueden eludir estas conductas, no tienen un "lugar donde esconderse", dado que están expuestos en cualquier momento a estas ciberconductas (Smith, 2013 p. 178). Si a ello añadimos la posibilidad de que un solo hecho dañino en la red pueda suponer una repetición constante en el tiempo (Vandebosch y Van Cleemput, 2009), aumenta el sentimiento de falta de control en la víctima (Navarro, Yubero y Larrañaga, 2018).

En cuanto a la prevalencia, se evidencia un aumento considerable de involucración en ciberbullying en la presente década (Kowalski, Limber y McCord, 2019). No obstante, se hace difícil establecer tasas de prevalencia, hallamos diferencias significativas debido a la diferente conceptualización del ciberbullying y los instrumentos utilizados para su medición (Ovejero, Yubero, Larrañaga y Moral, 2016; Zych et al., 2016). Respecto a la prevalencia media en cibervictimización, hallamos 
estudios que la sitúan entre el 20 y el 30\% (Brochado, Soares, y Fraga, 2017; Garaigordobil, 2011; Zych et al., 2016).

En cuanto a la prevalencia en ciber perpetración, se observa así mismo un aumento en los últimos años en la implicación de estas conductas por parte de los adolescentes (Aboujaoude et al., 2015; Rice et al., 2015). En un estudio de prevalencia realizado por Buelga, Iranzo, Cava, y Torralba (2015) en jóvenes de la Comunidad Valenciana (España), se constata una involucración en ciberagresión de un $56,5 \%$.

Atendiendo a la relación entre ciberbullying y sexo no hay consenso entre las diferentes investigaciones realizadas. Según la revisión de literatura científica, encontramos tres posturas diferentes. Así, una mayoría de estudios sitúan a las chicas como más victimizadas que los chicos (Aboujaoude et al., 2015, Buelga, Cava y Musitu, 2010; Baldry, Farrington y Sorrentino, 2015; Capadoccia, Craig y Pepler, 2013; Sourander et al, 2013; Stewart, Drescher, Maack, Ebesutani y Young, 2014). Por otro lado, otros autores sostienen que los chicos tienen mayor probabilidad de ser cibervictimizados que las chicas (Fanti, Demetriou, y Hawa, 2012; Pelfrey y Weber, 2013; Popovic-Citic, Djuric, y Cvetkovic 2011). Una tercera corriente caracteriza una serie de estudios (Fletcher, Fitzgerald-Yau, Jones, Allen, Viner y Bonell, 2014; Griezel, Finger, Bodkin-Andrews, Craven, y Yeung, 2012; Hinduja y Patchin, 2008; Smith et al., 2008) en las que no se encuentran diferencias significativas en cuanto a cibervictimización según el sexo de los adolescentes

Respecto a la relación entre las variables edad y ciberbullying, tampoco hay un acuerdo en los estudios efectuados. De igual manera que respecto a la relación entre ciberbullying y sexo, hallamos tres posturas preponderantes. Algunos autores sostienen un mayor porcentaje de víctimas de menor edad que los ciberagresores (Garaigordobil, 2015; Smith et al., 2008). Otros autores indican la existencia de una relación curvilínea entre edad y ciberbullying. De este modo, el mayor porcentaje de cibervíctimas se sitúa entre los 12 y los 14 años (Buelga et al., 2010; Tokunaga, 2010). Por último, otros autores, Jung et al. (2014) encuentran un mayor porcentaje de cibervíctimas en los últimos años de la adolescencia respecto a los primeros años de ésta.

Teniendo en cuenta estos antecedentes, Los objetivos del presente trabajo fueron: 1) Analizar la prevalencia de cibervictimización y ciberagresión en esta muestra de adolescentes; 2) Analizar si existen diferencias entre chicos y chicas en la cibervictimización y ciberagresión; y 3) Analizar si existen diferencias según el curso académico de los adolescentes $\left(1^{\circ}, 2^{\circ}, 3^{\circ}\right.$, y $4^{\circ}$ de la ESO) en la cibervictimización y ciberagresión.

\section{METODOLOGÍA}

\section{Participantes}

En el presente estudio participaron un total de 660 adolescentes de ambos sexos $(53.2 \%$ chicos y $46.8 \%$ chicas), de edades entre los 12 y los 17 años $(M=13.58, D T=1.26)$. Los participantes pertenecieron a tres institutos públicos de Educación Secundaria Obligatoria de la provincia de Valencia, y cursaban desde 1ํ de la ESO (Educación Secundaria Obligatoria) hasta 4ํㅜ de la ESO. En relación a la distribución de la muestra en función del curso académico, un 32.1\% ( $n=136)$ cursaban $1^{\circ}$ de la ESO, un $28.5 \%(n=121) 2^{\circ}$ de la ESO, un $21.2 \%(n=90) 3^{\circ}$ de la ESO y un $18.2 \%(n$ = 77) $4^{0}$ de la ESO.

\section{Instrumentos}

Cuestionario Sociodemográfico. Este cuestionario se compone de dos preguntas: una para que el adolescente indique el curso académico al que pertenece, y otra para el adolescente indique si es chico 0 chica. 


\section{CYBERBULLYING: DIFERENCIAS EN FUNCIÓN DEL SEXO Y CURSO ACADÉMICO}

Escala de Victimización a través del Teléfono Móvil y de Internet (CYBVIC; Buelga et al., 2010). Esta escala se compone de 10 ítems que evalúan la experiencia del adolescente como víctima de acoso a través del móvil o Internet en el último año (por ejemplo: "Me han amenazado para meterme miedo"). Se trata de una escala tipo Likert con opciones de respuesta desde 1 (nunca) a 4 (siempre). Esta escala contiene además un ítem para conocer la duración de la victimización a través de las tecnologías (no me ha pasado, 1 mes (o menos), entre 3 y 6 meses, o 1 año (o más)). El coeficiente de fiabilidad alpha de Cronbach de la escala fue de 0.81 .

Escala de Agresión a través del Teléfono Móvil y de Internet (CYBAG; Buelga et al., 2010). Esta escala se compone de 10 ítems que evalúan la experiencia del adolescente como agresor de acoso a través del móvil o Internet en el último año (por ejemplo: "He enviado cosas de miedo o cosas guarras a alguien para asustarla o fastidiarle"). Se trata de una escala tipo Likert con opciones de respuesta desde 1 (nunca) a 4 (siempre). Esta escala contiene además un ítem para conocer la duración de la victimización a través de las tecnologías (no me ha pasado, 1 mes (o menos), entre 3 y 6 meses, 01 año (o más)). El coeficiente de fiabilidad alpha de Cronbach de la escala fue de 0.82.

\section{Procedimiento}

En primer lugar, se realizaron sesiones de información en los centros educativos participantes para explicar al personal docente los propósitos de esta investigación. Posteriormente, una vez obtenidos los permisos correspondientes y las autorizaciones paternas en los centros educativos, investigadores entrenados pasaron los diferentes instrumentos de este estudio a los adolescentes. El pase de cuestionarios a los adolescentes fue voluntario y anónimo.

\section{Análisis de datos}

El análisis de los datos de esta investigación se realizó con el programa estadístico SPSS, versión 22. Previo a los objetivos de la investigación, en primer lugar, se realizaron análisis de fiabilidad para comprobar la consistencia interna de cada una de las escalas utilizadas. En segundo lugar, a través de un análisis de frecuencia se calculó la prevalencia de cibervictimización y ciberagresión en los adolescentes participantes. Se realizó una prueba $T$ para comprobar si existen diferencias entre chicos y chicas tanto en la cibervictimización, como en la ciberagresión. En tercer lugar, se llevó a cabo un análisis de varianza (ANOVA de un factor) para analizar si existen diferencias en el cyberbullying (victimización y agresión) en función del curso académico (1ํㅜ는 $3^{\circ}$, y $4^{\circ}$ de la ESO). También se aplicó la prueba post-hoc de Bonferroni, para conocer exactamente entre que cursos académicos están las diferencias.

\section{RESULTADOS}

En primer lugar, los resultados muestran que del total de los adolescentes que participaron en esta investigación, un $71.5 \%$ de los adolescentes no habían sido victimizados a través de las tecnologías. Por el contrario, un $28.5 \%$ de los adolescentes sí que habían sido víctimas de cyberbullying.

Por lo que se refiere a la ciberagresión, un $66.5 \%$ de los adolescentes no habían sido agresores de cyberbullying, mientras que un $33.5 \%$ de los participantes sí que agredieron a sus compañeros a través de las tecnologías.

Para calcular estos resultados, se especificaron como cibervíctimas o ciberagresores aquellos adolescentes que obtuvieron una puntuación superior o igual a la media más una desviación típica en cada una de estas dos variables.

Por lo que se refiere a los propósitos de esta investigación, respecto a las diferencias en la cibervictimización y la ciberagresión en función del sexo, los resultados no hallan diferencias significati- 
vas entre los grupos en estas variables. En concreto, no se observan diferencias significativas entre chicos y chicas en la variable cibervictimización ( $T=1.194, p=.275)$. Sin embargo, tampoco se observan diferencias significativas entre chicos y chicas en la variable cibervictimización ( $T=.005$, $\mathrm{p}=.942)$.

Tabla 1. Diferencia de medias en cibervictimización y ciberagresión en función del sexo (chicos y chicas)

\begin{tabular}{lccc}
\hline & Chicos & Chicas & $T$ \\
\hline Variables & & & \\
\hline Cibervictimización & 1.25 & 1.29 & 1.19 \\
Ciberagresión & $(0.40)$ & $(0.41)$ & \\
& 1.21 & 1.22 & .005 \\
\hline & $(0.31)$ & $(0.29)$ & \\
\hline
\end{tabular}

Nota: Medias (desviaciones típicas)

Por lo que respecta a las diferencias en la cibervictimización y la ciberagresión en función del curso académico, los resultados evidencian que no hay diferencias entre los cursos académicos en la cibervictimización ( $F=2.42, p=0.65)$, pero si en la ciberagresión.

Tabla 2. Diferencia de medias en cibervictimización y ciberagresión en función del curso académico $\left(1^{\circ}, 2^{\circ}, 3^{\circ}\right.$, y $4^{\circ}$ de la ESO)

$1^{\circ} \mathrm{ESO} \quad 2^{\circ} \mathrm{ESO} \quad 3^{\circ} \mathrm{ESO} \quad 4^{\circ} \mathrm{ESO} \quad F$

\begin{tabular}{lccccc}
\hline Variables & \multicolumn{7}{c}{} & & \\
\hline & 1.25 & 1.26 & 1.34 & 1.22 & 2.42 \\
Cibervictimización & $(0.40)$ & $(0.37)$ & $(0.46)$ & $(0.35)$ & \\
Ciberagresión & $1.15^{\mathrm{b}}$ & 1.21 & $1.27^{\mathrm{a}}$ & $1.25^{\mathrm{a}}$ & $4.65^{*}$ \\
& $(0.20)$ & $(0.29)$ & $(0.34)$ & $(0.37)$ & \\
\hline
\end{tabular}

Nota: Medias (desviaciones típicas); Bonferroni: $a>b>c$; ${ }^{*} p<0.05$

En concreto, los resultados muestran que hay diferencias en la ciberagresión en función de los diferentes cursos académicos al que pertenecen los adolescentes ( $F=4.65, p=0.03$ ). De forma específica, en la prueba post-hoc de Bonferroni se evidencia que los adolescentes que pertenecen a $3^{0}$ y $4^{0}$ de la ESO tienen puntuaciones en ciberagresión significativamente superiores al grupo de adolescentes de $1^{0}$ de la ESO.

\section{CONCLUSIONES}

El objetivo de este estudio fue en primer lugar, analizar la prevalencia de adolescentes víctimas y agresores de acoso a través de las tecnologías en una muestra de 660 adolescentes. Los resultados obtenidos muestran que la prevalencia de víctimas de cyberbullying en nuestro estudio fue del 28.5\%. Esta prevalencia está en consonancia con el estudio de Floros et al., (2013). Sin embargo, 


\section{CYBERBULLYING: DIFERENCIAS EN FUNCIÓN DEL SEXO Y CURSO ACADÉMICO}

supera el promedio actual de prevalencia que suelen hallar otros estudios. Por ejemplo, Hinduja y Patchin (2012), señalan una prevalencia de cibervictimización de un $20 \%$. A su vez, en un meta-análisis sobre 80 estudios internacionales (Modecki, Minchin, Harbaugh, Guerra y Runions, 2014) reportaban una tasa de un $15 \%$ de cibervictimización. Por otro lado, otros estudios (Álvarez-García, Núñez, Dobarro y Rodríguez, 2015) obtienen una prevalencia superior a la hallada en nuestro estudio, en concreto estos autores informan de una prevalencia de víctimas de cyberbullying del $78 \%$. Estos resultados pueden deberse a la enorme expansión y alta disponibilidad de las tecnologías de los últimos años (Brochado et al., 2017).

Por lo que se refiere a la prevalencia de ciberagresores, nuestros resultados muestran una prevalencia del $33.5 \%$ de adolescentes que han agredido a algún compañero a través de las tecnologías. Este resultado está en consonancia con el estudio de Lee y Shin (2017), que obtiene una prevalencia del $31,5 \%$. Sin embargo, encontramos otros estudios donde se señala una prevalencia inferior, por ejemplo, en el estudio de Del Rey et al. (2015), que refiere una tasa de ciberagresión de un $5,12 \%$ y por otra parte, estudios con un porcentaje superior en cuanto a conductas de ciberagresión, como el de Buelga, Iranzo, Cava y Torralba (2015) que señalan una prevalencia de ciberagresión del $56,5 \%$. La diferencia entre las prevalencias en función de los diferentes estudios se debe a la variedad cultural de cada muestra, y a la utilización de diferentes metodologías y cuestionarios para medir el cyberbullying (Thomas, Connor y Scott (2015).

En segundo lugar, el objetivo de este estudio fue analizar si existen diferencias entre chicos y chicas en la cibervictimización y ciberagresión. Los resultados obtenidos muestran que no se observan diferencias significativas entre chicos y chicas en la variable cibervictimización, ni en la ciberagresión. Estos resultados están en la línea de los obtenidos por Fletcher et al. (2014). En concreto estos autores no encuentran diferencias entre chicos y chicas en el cyberbullying. Sin embargo, existen otras investigaciones donde sí que observan diferencias en el cyberbullying en función del sexo. La investigación de Walrave y Heirman (2011), muestra que las chicas suelen ser más víctimas, y los chicos más agresores. Por otro lado, el estudio de Mark y Ratliffe (2011) muestra que las chicas se muestran más involucradas que los chicos, tanto como ciberagresores como cibervíctimas. Diversos autores, señalan que es conveniente estudiar las diferentes modalidades de cyberbullying teniendo en cuenta el sexo de los adolescentes, para poder extraer así más información respecto a la ciberconducta de chicos y chicas (Álvarez, Barreiro-Collazo y Nuñez, 2017; Vandebosch y Van Cleemput, 2009).

En tercer lugar, el propósito de este estudio fue Analizar si existen diferencias según el curso


Por un lado, nuestros resultados muestran que no hay diferencias entre los cursos académicos en la cibervictimización. Este resultado está en coherencia al estudio de Ortega Ruiz, Calmaestra Villén y Mora Merchán (2008) que muestra que no hay diferencias en la cibervíctimas en función de la edad. Así parece que, en cualquier curso académico pueda aparecer el cyberbullying. Por otro lado, nuestros resultados muestran que los adolescentes que pertenecen a $3^{\circ}$ y $4^{\circ}$ de la ESO tienen puntuaciones en ciberagresión significativamente superiores al grupo de adolescentes de 1ํ de la ESO. En este sentido, Garaigordobil (2015) evidencia que los adolescentes más mayores son los que suelen intimidar a los más pequeños. De esta forma, los ciberagresores más mayores suelen ciberintimidar a los compañeros de cursos inferiores.

Finalmente debemos señalar que esta investigación cuenta con determinadas limitaciones que hay que tener en cuenta. En primer lugar, al tratarse de un estudio transversal, no nos permite establecer relaciones de causalidad entre las variables objeto de estudio. Para futuras investigaciones, sería interesante establecer un diseño longitudinal que nos permita conocer las diferencias en con- 
ductas de ciberagresión y cibervictimización según sexo y edad. Por otro lado, otra limitación de este estudio es que se trata de una investigación únicamente cuantitativa. Sería conveniente en un futuro implementar un diseño de investigación complementario cuantitativo-cualitativo que nos permita profundizar en la experiencia del cyberbullying mediante entrevistas 0 grupos focales.

A pesar de estas limitaciones, los hallazgos de la presente investigación aportan datos interesantes para una mejor comprensión de las diferencias en cuanto la involucración de chicos y chicas en conductas de ciberagresión y cibervictimización.

\section{REFERENCIAS BIBLIOGRÁFICAS}

Aboujaoude, E., Savage, M. W., Starcevic, V., y Salame, W. O. (2015). Cyberbullying: Review of an old problem gone viral. Journal of Adolescent Health, 57(1), 10-18.

Álvarez-García, D., Barreiro-collazo, A., y Núñez, J. (2017). Cyberaggression among Adolescents: Prevalence and Gender Differences / Ciberagresión entre adolescentes: prevalencia y diferencias de género. Comunicar, 25(50), 89-97.

Álvarez-García, D., Núñezez, J. C., Dobarro, A., y Rodríguez, C. (2015). Risk factors asso-ciated with cybervictimization in adolescence. International Journal of Clinicaland Health Psychology, 15, 226-235.

Baldry, A. C., Farrington, D. P., \& Sorrentino, A. (2015). "Am I at risk of cyberbullying"? A narrative review and conceptual framework for research on risk of cyberbullying and cybervictimization: The risk and needs assessment approach. Aggression and Violent Behavior, 23, 36-51.

Betts, Lucy R., and Karin A. Spenser. 2017. "People think it's a harmless joke": Young people's understanding of the impact of technology, digital vulnerability and cyberbullying in the United Kingdom. Journal of Children and Media 11: 20-35.

Brochado, S., Soares, S. y Fraga, S. (2017). A scoping review on studies of cyberbullying prevalence among adolescents. Trauma, Violence, \& Abuse, 18(5), 523-531.

Buelga, S., Cava, M. J. y Musitu, G. (2010). Cyberbullying: Victimización entre adolescentes a través del teléfono móvil y de Internet. Psicothema, 22(4),784-789.

Buelga, S., Iranzo, B., Cava, M. J., y Torralba, E. (2015). Perfil psicosocial de adolescentes agresores de cyberbullying. Revista de Psicología Social, 30(2), 382-406.

Buelga, S., y Pons, J. (2011). Aggressions among adolescents through mobile phones and the Internet. Psychosocial Intervention, 21(1), 91-101.

Cappadocia, M.C., Craig, W.M. y Pepler, D., 2013. Cyberbullying: prevalence, stability, and risk factors during adolescence. Can. J. Sch. Psychol. 28 (2), 171-192. Del Rey, R., Casas, J. A., OrtegaRuiz, R., Schultze-Krumbholz, A., Scheithauer, H., Smith, P., y Plichta, P. (2015). Structural validation and cross-cultural robustnessof the European Cyberbullying Intervention Project Questionnaire. Computers in Human Behavior, 50, 141-147.

Fanti, K. A., Demetriou, A. G., \& Hawa, V. V. (2012). A longitudinal study of cyberbullying: Examining riskand protective factors. European Journal of Developmental Psychology, 9(2), 168-181. doi:10.1080/17405629.2011.643169.

Fletcher, A., Fitzgerald-Yau, N., Jones, R., Allen, E., Viner, R. M., \& Bonell, C. (2014). Brief report: Cyberbullying perpetration and its associations with socio demographics, aggressive behaviour at school, and mental health outcomes. Journal of Adolescence, 37(8), 1393-1398.

Floros, Georgios D., Konstantinos E. Siomos, Virginia Fisoun, Evaggelia Dafouli, and Dimitrios Geroukalis. 2013. Adolescent Online Cyberbullying in Greece: The impact of parental online security practices, bonding, and online impulsiveness. Journal of School Health 83: 445-53.

Garaigordobil, M. (2015). Ciberbullying en adolescentes y jóvenes del País Vasco: Cambios con 


\section{CYBERBULLYING: DIFERENCIAS EN FUNCIÓN DEL SEXO Y CURSO ACADÉMICO}

la edad. Anales de psicología, 31(3), 1069-1076.

Garaigordobil, M. (2011). Prevalencia y consecuencias del cyberbullying: una revisión. International Journal of Psychological Therapy, 11(2), 233-254.

Garaigordobil, M. y Martinez-Valderrey, V. (2014). Programa de intervencion para prevenir y reducir el ciberbullying. Larousse - Ediciones Piramide. Madrid.

Giumetti, G. W., y Kowalski, R. M. (2016). Cyberbullying matters: Examining the incremental impact of cyberbullying on outcomes over and above traditional bullying in North America. En R. Navarro, S. Yubero, y E. Larrañaga (Eds.), Cyberbullying across the Globe (pp. 117-130). Madrid: Springer International Publishing.

Griezel, L., Finger, L. R., Bodkin-Andrews, G. H., Craven, R. G., y Yeung, A. S. (2012). Uncovering the structure of gender and developmental differences in cyber bullying. The Journal of Educational Research, 105(6), 442-455.

Hinduja S y Patchin JW (2008). Cyberbullying: an exploratory analysis of factors related to offending and victimization. Deviant Behavior, 29, 129-156.

Hinduja, S., y Patchin, J. W. (2012). Cyberbullying: Neither an epidemic nor a rarity? European Journal of Developmental Psychology, 9, 539-543.

Kowalski, R. M., Limber, S. P., y McCord, A. (2019). A developmental approach to cyberbullying: Prevalence and protective factors. Aggression and Violent Behavior, 20-32.

Lee, Changho, and Namin Shin. 2017. Prevalence of cyberbullying and predictors of cyberbullying perpetration among korean adolescents. Computers in Human Behavior 68: 352-58.

Mark, L., y Ratliffe, K. T. (2011). Cyber worlds: New playgrounds for bullying. Computers in Schools, 28, 92-116.

Modecki, K. L., Minchin, J., Harbaugh, A. G., Guerra, N. G., y Runions, K. C. (2014). Bullying prevalence across contexts: A meta-analysis measuring cyber and traditional bullying. Journal of Adolescent Health,

Navarro, R., Yubero, S., y Larrañaga, E. (2018). Cyberbullying victimization and fatalism in adolescence: Resilience as a moderator. Children and Youth Services Review, 84, 215-221.

Olweus, D. (1998). Conductas de acoso y amenaza entre escolares. Madrid: Ediciones Morata.

Ortega-Barón, J., y Carrascosa, L. (2018). Malestar psicológico y apoyo psicosocial en víctimas de ciberbullying. International Journal of Developmental and Educational Psychology. Revista INFAD de Psicología., 2(1), 357.

Ortega Barón, J., Postigo, J., Iranzo, B., Buelga, S., y Carrascosa, L. (2018). Parental Communication and Feelings of Affiliation in Adolescent Aggressors and Victims of Cyberbullying. Social Sciences, 8(1), 3. Ortega Ruiz, R., Calmaestra Villén, J., y Mora Merchán, J. A. (2008). Cyberbullying. International Journal of Psychology and Psychological Therapy, 8 (2), 183-192.

Ovejero, A., Yubero, S., Larrañaga, E. y Moral M.V. (2016). Cyberbullying: Definitions and Facts from a Psychosocial Perspective. En R. Navarro, S. Yubero y E. Larrañaga (Eds). Cyberbullying Across the Globe: Gender, Family, and Mental Health (pp. 1-31). Prólogo de Peter K. Smith. Springer.

Patchin, J. W., e Hinduja, S. (2013). Cyberbullying Among Adolescents: Implications for Empirical Research. Journal of Adolescent Health, 53(4), 431-432.

Pelfrey, W. V., \& Weber, N. L. (2013). Keyboard gangsters: analysis of incidence and correlates of cyberbullying in a large urban student population. Deviant Behavior, 34(1), 68-84.

Popovic-Citic, B., Djuric, S., \& Cvetkovic, V. (2011). The prevalence of cyberbullying among adolescents: A case study of middle schools in Serbia. School Psychology International, 32, 412-424. 
Pornari, C. D., y Wood, J. (2010). Peer and cyber agression in secondary school students: The role of moral disengagement, hostile attribution bias, and outcome expectancies. Aggressive Behaviour, 36(2), 81-94.

Rice, E., Petering, R., Rhoades, H., Winetrobe, H., Goldbach, J., Plant, A.,...Kordic, T. (2015). Cyberbullying perpetration and victimization among middle-school students. American Journal of Public Health, 105(3), 66-72.

Rodríguez-Álvarez, J. M., Cabrera-Herrera, M. del C., y Yubero Jiménez, S. (2018). Los riesgos de las TIC en las relaciones entre iguales. Cyberbullying en Educación Primaria y Secundaria. Innoeduca. International Journal of Technology and Educational Innovation, 4(2), 185.

Thomas, H. J., Connor, J. P., \& Scott, J. G. (2015). Integrating traditional bullying and cyberbullying: Challenges of definition and measurement in adolescents-A review. Educational Psychology Review, 27(1), 135-152.

Smith, P.K. (2013). Cyberbullying y ciberagresión. En A. Ovejero, P. Smith y S.Yubero (Eds.) El acoso escolar y su prevención:cperspectivas internacionales (pp. 173-189). Madrid. Biblioteca Nueva.

Smith, P. K. (2019). Research on Cyberbullying: Strengths and Limitations. En: Vandebosch H., Green L. (eds) Narratives in Research and Interventions on Cyberbullying among Young People. Springer International Publishing.

Smith, P. K., Mahdavi, J.M., Fisher, S., Russell, S., y Tippett, N. (2008). Cyberbullying: It's nature and impact in secondary school pupils. Journal of Child Psychology and Psychiatry, 49(4), 376-385

Sourander, A., Klomek, A. B., Ikonen, M., Lindroos, J., Luntamo, T., Koskelainen, M.,... Helenius, H. (2010). Psychosocial risk factors associated with cyberbullying among adolescents. Archives of General Psychiatry, 67, 720-728.

Stewart, R. W., Drescher, C. F., Maack, D. J., Ebesutani, C., y Young, J. (2014). The development and psychometric investigation of the Cyberbullying Scale. Journal of Interpersonal Violence, 29(12), 2218-2238.

Tokunaga, R. S. (2010). Following you home from school: A critical review and synthesis of research on cyberbullying victimization. Computers and Human Behavior, 26, 277-287.

Vandebosch, H., y Van Cleemput, K. (2009). Cyberbullying among youngsters: profiles of bullies and victims. New Media \& Society, 11(8), 1349-1371.

Walrave, M., y Heirman, W. (2011). Cyberbullying: Predicting victimization and perpetration. Children \& Society, 25(1), 59-72.

Zych, I., Ortega-Ruiz, R., \& Marín-López, I. (2016). Cyberbullying: a systematic review of research, its prevalence and assessment issues in Spanish studies. Psicología Educativa, 22(1), 5-18. 
